\title{
Subgap structures in the current-voltage characteristic of the intrinsic Josephson effect due to phonons
}

\author{
Ch. Helm, Ch. Preis, F. Forsthofer and J. Keller, \\ Institut für Theoretische Physik, Universität Regensburg, D-93040 Regensburg, Germany \\ K. Schlenga, R. Kleiner, and P. Müller, \\ Physikalisches Institut III, Universität Erlangen-Nürnberg, D-91058 Erlangen, Germany
}

(October 4, 2018)

\begin{abstract}
A modified RSJ-model for the coupling of intrinsic Josephson oscillations and c-axis phonons in the high- $T_{c}$ superconductors $\mathrm{Tl}_{2} \mathrm{Ba}_{2} \mathrm{Ca}_{2} \mathrm{Cu}_{3} \mathrm{O}_{10+\delta}$ and $\mathrm{Bi}_{2} \mathrm{Sr}_{2} \mathrm{CaCu}_{2} \mathrm{O}_{8+\delta}$ is developed. This provides a very good explanation for recently reported subgap structures in the $I$ - $V$-characteristic of the caxis transport. It turns out that the voltages of these structures coincide with the eigenfrequencies of longitudinal optical phonons, providing a new measurement technique for this quantity. The significantly enhanced microwave emission at the subgap structures in both the $\mathrm{GHz}$ and $\mathrm{THz}$ region is discussed.
\end{abstract}

The intrinsic Josephson effect between the $\mathrm{CuO}_{2}$ (multi)-layers in highly anisotropic cuprate superconductors has been demonstrated in a characteristic series of branches in the current-voltage characteristic in c-direction [1]. Each branch corresponds to a well defined number of Josephson junctions in the resistive state. Recently the observation of subgap structures in the $I$ - $V$-characteristic of $\mathrm{Tl}_{2} \mathrm{Ba}_{2} \mathrm{Ca}_{2} \mathrm{Cu}_{3} \mathrm{O}_{10+\delta}$ (TBCCO) and $\mathrm{Bi}_{2} \mathrm{Sr}_{2} \mathrm{CaCu}_{2} \mathrm{O}_{8+\delta}$ (BSCCO) has been reported [2, 3]. The structures in the different branches can be traced back to the $I$ - $V$-characteristic of one single Josephson junction in the resistive state. These structures seem to be an intrinsic effect, as they have been observed in junctions of different geometries. Furthermore the characteristic voltages of the subgap structures are completely independent of temperature and external magnetic fields, which rules out any relation to the superconducting gap, vortex flow or the thermal excitation of quasiparticles.

In this paper we will show that these subgap structures can very well be explained by the coupling of c-axis phonons to the Josephson field oscillations in a single resistive junction. Thereby it turns out that the maxima in the $I-V$-characteristic correspond to the eigenfrequencies of longitudinal lattice vibrations. A more detailed discussion can be found in [4].

In the RSJ model the total current density through a junction

$$
I / F=j_{c} \sin \gamma+j_{q p}(E)+\dot{D}
$$

is the sum of the Josephson current, the quasiparticle current and the displacement current density. The (gauge invariant) phase difference $\gamma$ is related to the electric field $E$ in the barrier of thickness $b$ by the Josephson relation

$$
\hbar \dot{\gamma}=2 e E b .
$$

In the simplest case the quasiparticle current density is described by an ohmic law $j_{q p}=\sigma E$. Here we will use a more general form $j_{q p}(E)=j_{q p}(\bar{E})+\sigma(E(t)-\bar{E})$ with $\sigma=j_{q p}^{\prime}(\bar{E})$ by expanding the field $E(t)$ around the time averaged field $\bar{E}$. The displacement current density
$\dot{D}$ contains the polarization $P$ of the barrier medium, $D=\epsilon_{0} E+P=\epsilon_{0} \epsilon E$. In the case of high frequency Josephson oscillations in the range of phonon frequencies it is important to keep the full frequency dependence of $\epsilon(\omega)$ or to treat the polarization $P$ as an additional dynamical variable.

Here we assume that the polarization $P$ is due to a c-axis displacement $z$ of ions with charge $q$, mass $M$ and density $n$ in the non superconducting barrier between the copper oxide (multi)-layers. For the motion of the ions we assume a simple oscillator

$$
\ddot{z}+\omega_{0}^{2} z+\rho \dot{z}=\frac{q}{M} E,
$$

which is driven by the electric field $E$ in the barrier. In this model the contribution of the oscillator to the dielectric function is given by

$$
\epsilon(\omega)=\epsilon_{\infty}+\frac{S \omega_{0}^{2}}{\omega_{0}^{2}-\omega^{2}-i \rho \omega}
$$

where $S=n q^{2} /\left(\epsilon_{0} M \omega_{0}^{2}\right)$ is the oscillator strength of the phonon and $\epsilon_{\infty}$ includes the other contributions to the polarizability of the barrier. In this form it is straightforward to generalize the calculation to several phonon branches.

The system is characterized by the transversal optical phonon frequency $\omega_{0}$, the Josephson plasma freqency $\omega_{J}^{2}=2 e b j_{c} /\left(\hbar \epsilon_{0} \epsilon_{\infty}\right)$ and a characteristic frequency $\omega_{c}=(2 e / \hbar) V_{c}, V_{c}$ being the voltage at the critical current $j_{c}$. In our case $\omega_{J}<\omega_{0}<\omega_{c}$ and the McCumber parameter $\beta_{c}=\omega_{c}^{2} / \omega_{J}^{2}$ is large.

Using equ. (2) in (1) and (3) we obtain a coupled set of differential equations for the phase $\gamma$ and the polarization amplitude $z$, which can be solved numerically. Then the time average of the phase velocity $\langle\dot{\gamma}\rangle=(2 e / \hbar) V$ determines the dc voltage $V$ shown in the $I-V$ characteristic.

The most important features of the model can also be understood analytically: Due to the large McCumber parameter $\beta_{c}$ both the phase and the polarization

$$
\begin{aligned}
& \gamma(t) \approx \gamma_{0}+\omega t+\gamma_{1} \sin \omega t, \\
& z(t) \approx z_{0}+z_{1} \cos (\omega t+\varphi) .
\end{aligned}
$$


oscillate primarily with the basic frequency $\omega=(2 e / \hbar) V$. It is also well known that in this case $\gamma_{1} \ll 1$ and $\sin (\gamma(t))$ can be expanded in orders of $\gamma_{1}$ keeping only the leading Fourier components:

$$
\sin \gamma(t) \approx \sin \gamma_{0} \cos \omega t+\cos \gamma_{0} \sin \omega t-\frac{1}{2} \gamma_{1} \sin \gamma_{0}
$$

Since the equation of motion for the phonon is linear, we can relate the phonon amplitude to the driving force $\dot{\gamma}$ by the phonon response function of equ. (41) at the frequency $\omega$. Thus we obtain for the coefficents of $\sin \omega t$, $\cos \omega t$ and the dc current $i=I / I_{c}$ :

$$
\begin{aligned}
& 0=\cos \gamma_{0}+\frac{\omega^{2}}{\omega_{J}^{2}} \epsilon_{1} \gamma_{1}, \\
& 0=\sin \gamma_{0}+\frac{\omega^{2}}{\omega_{J}^{2}}\left(\epsilon_{2}+\frac{\sigma}{\epsilon_{0} \omega}\right) \gamma_{1}, \\
& i=i_{q p}(V)-\frac{1}{2} \gamma_{1} \sin \gamma_{0},
\end{aligned}
$$

where $\epsilon_{1}, \epsilon_{2}$ are the real and imaginary part of the phonon dielectric function. From these three equations we finally obtain for the normalized dc-current

$$
\begin{aligned}
i(V) & =i_{q p}(V)+\frac{1}{2} \frac{\omega_{J}^{2}}{\omega^{2}} \frac{\epsilon_{2}+\frac{\sigma}{\omega \epsilon_{0}}}{\epsilon_{1}^{2}+\left(\epsilon_{2}+\frac{\sigma}{\omega \epsilon_{0}}\right)^{2}} \\
& =i_{q p}(V)-\frac{1}{2} \frac{\omega_{J}^{2}}{\omega^{2}} \operatorname{Im}\left(\frac{1}{\tilde{\epsilon}(\omega)}\right)
\end{aligned}
$$

with $\tilde{\epsilon}=\epsilon_{1}+i\left(\epsilon_{2}+\sigma /\left(\omega \epsilon_{0}\right)\right)$ and $\omega=(2 e / \hbar) V$.

This result clearly shows that maxima in the $I-V$ characteristic occur near the zeros of the phonon dielectric function $\epsilon_{1}$ exactly at the eigenfrequencies of longitudinal optical phonons with wavevector $\vec{q}=0$. Here the polarization and the Josephson field oscillations are compensating each other, and the energy transfer to the phonon is maximal. In contrast to this, the Josephson oscillation amplitude $\gamma_{1}$ has a minimum at the transversal optical eigenfrequency $\omega_{0}$.

In figures (1) and (2) this theory is compared with experimental results [3] by choosing appropriate values for the McCumber parameter $\beta_{c}$, the phonon frequencies $\omega_{0, i}$, their oscillator strength $S_{i}$ and damping $\rho_{i}$, and the quasi-particle characteristic. We see that the essential features are very well reproduced. We also find an excellent agreement between the numerical integration and the analytical results. Note that both in experiment and theory hysteretic regions appear near the phonon resonances.

In TBCCO the characteristic voltage is given by $V_{c}=$ $27.1 \mathrm{mV}$ and the two maxima correspond to LO-phonon frequencies $\nu_{\mathrm{LO}, 1}=3.65 \mathrm{THz}$ and $\nu_{\mathrm{LO}, 2}=4.70 \mathrm{THz}$. The corresponding values for BSCCO are: $V_{c}=21.8 \mathrm{mV}$, $\nu_{\mathrm{LO}, 1}=2.96 \mathrm{THz}, \nu_{\mathrm{LO}, 2}=3.90 \mathrm{THz}$ and $\nu_{\mathrm{LO}, 3}=$ $5.71 \mathrm{THz}$. These values are compatible with phonon resonances observed in the infrared reflectivity at $3.7 \mathrm{THz}$ and $4.3 \mathrm{THz}$ of polycristalline samples of TBCCO [6]. Unfortunately for both TBCCO and BSCCO no reliable infrared experiments for c-axis phonons in single crystals are available [6.7]. Lattice dynamical calculations [8] show phonon modes with large oscillator strength, where $\mathrm{TlO}$ layers are oscillating against $\mathrm{CuO}$ layers at somewhat higher frequencies. The dispersion of these LO-phonons in c-direction is small, which causes a small, but finite damping $\rho$ in the model described above.

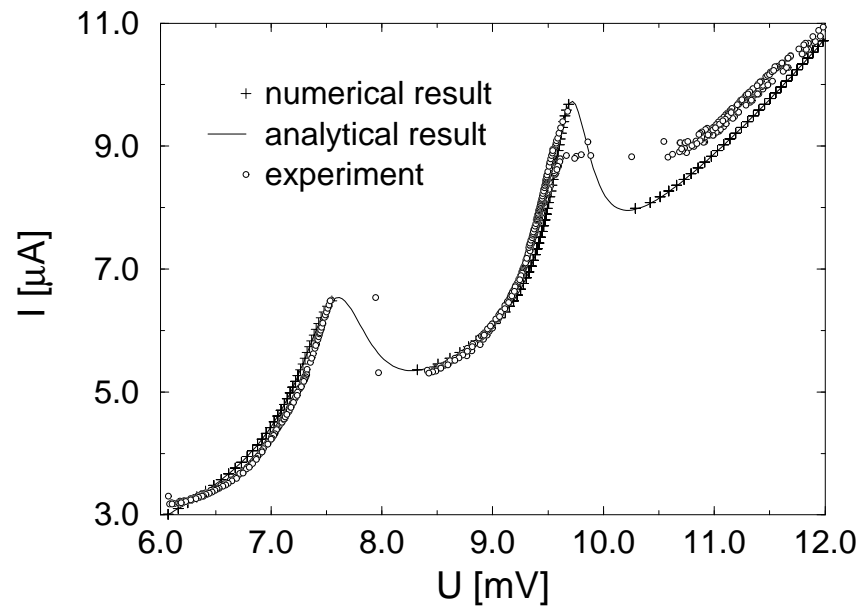

FIG. 1. Experimental, analytical and numerical $I-V$-characteristic of TBCCO for $\beta_{c}=375, \omega_{0,1}=3.28 \mathrm{THz}$, $S_{1}=2.87, \rho_{1}=0.2 \mathrm{THz}, \omega_{0,2}=4.45 \mathrm{THz}, S_{2}=0.68$, $\rho_{2}=0.4 \mathrm{THz}, \epsilon_{\infty}=8.45$.

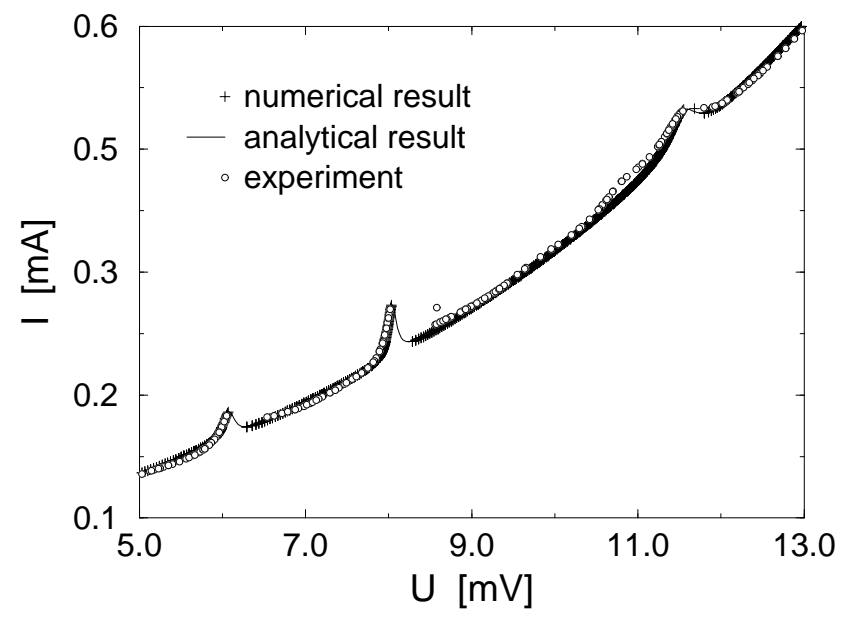

FIG. 2. Experimental, analytical and numerical $I$ - $V$-characteristic of $\mathrm{BSCCO}$ for $\beta_{c}=800, \omega_{0,1}=2.80 \mathrm{THz}$, $S_{1}=1.35, \rho_{1}=0.08 \mathrm{THz}, \omega_{0,2}=3.62 \mathrm{THz}, S_{2}=1.5$, $\rho_{2}=0.05 \mathrm{THz}, \omega_{0,3}=4.91 \mathrm{THz}, S_{3}=1.65, \rho_{3}=0.2 \mathrm{THz}$, $\epsilon_{\infty}=7.5$.

A similar calculation can be used to determine the microwave power radiated by Josephson oscillations: the emission is enhanced by about one order of magnitude near the maxima of the subgap structures, suggesting these voltages for an experimental attempt to demonstrate the microwave emission of the intrinsic Josephson oscillations directly. Also the observed enhancement of 
the microwave emission in the $\mathrm{GHz}$ region, if the junction is biased near the subgap structures, can be explained: the vanishing differential conductivity near the maxima of the $I-V$-curve leads to an amplification of voltage fluctuations caused by thermal fluctuations of the current.

Further theoretical investigations suggest that also the mixing of two external frequencies (in the $\mathrm{THz}$ region) down to the difference frequency $(\mathrm{GHz})$ is much more efficient, if the external frequencies are near the maxima of the subgap structures.

The modifications of the intrinsic Josephson oscillations through the dielectric constant of the phonons is in complete formal analogy with the coupling of a macroscopic Josephson junction to an external electromagnetic system with a resonance, i.e. a cavity. In such systems hysteretic structures in the $I-V$-characteristic, which correspond to the subgap structures considered in this publication, have been reported repeatedly [5].

To summarize, the recently discovered subgap structures in the $I-V$-characteristic in the intrinsic Josephsoneffect in high- $T_{c}$-superconductors are explained by a coupling of Josephson oscillations to optical c-axis phonons within a modified RSJ-model. Apart from perfect reproduction of the experimental data for both BSCCO and TBCCO new insights in the physical interpretation of the peak structures are obtained. The peak position is identified with the eigenfrequency of the longitudinal optical phonon, which provides a new possibility of a direct measurement of this quantity, which is normally hard to determine in optical experiments. Significantly enhanced microwave emission near the maxima of the subgap structure in the $\mathrm{GHz}$ region is explained and theoretically predicted in the $\mathrm{THz}$ region.

Acknowledgment: This work has been supported by a grant of the Bayerische Forschungsstiftung within the research program FORSUPRA and by the Studienstiftung des Deutschen Volkes (C.H.).

[1] R. Kleiner, F. Steinmeyer, G. Kunkel, and P. Müller, Phys. Rev. Lett. 68, 2394 (1992); R. Kleiner, and P. Müller, Phys. Rev. B 49, 1327 (1994).

[2] A. Yurgens, D. Winkler, N. Zavaritsky, and T. Claeson, SPIE Proceedings, Conference on Oxide Superconductor Physics and Nano-Engineering II, vol. 2697 (1996).

[3] K. Schlenga, G. Hechtfischer, R. Kleiner, W. Walkenhorst, P. Müller, H.L. Johnson, M. Veith, W. Brodkorb, and E. Steinbeiß, Phys. Rev. Lett. 76, 4993 (1996).

[4] F.Forsthofer, Ch. Helm, Ch. Preis, J. Keller, K. Schlenga, R. Kleiner, and P. Müller, cond-mat/9701049, submitted to Phys. Rev. Letters.

[5] Konstantin K. Likharev "Dynamics of Josephson Junctions and Circuits", Gordon and Breach 1996, chapter 12 and references therein; K. Baberschke, K.D. Bures, S.E. Barnes, Phys. Rev. Lett. 52, 98 (1984).

[6] T. Zetterer, M. Franz, J. Schützmann, W. Ose, H.H. Otto, and K.F. Renk, Phys. Rev. B 41, 9499 (1990); V.M. Burlakov, S.V. Shulga, J. Keller, and K.F. Renk, Physica C, 203, 68 (1992) and references therein.

[7] R. G. Buckley, M.P. Staines, D.M. Pooke, T. Stoto, and N.E. Flower, Physica C 248, 247 (1995) and references therein.

[8] J. Prade, A.D. Kulkarni, F.W. de Wette, U. Schröder, and W. Kress, Phys. Rev. B 39, 2771 (1989); A. D. Kulkarni, F.W. de Wette, J. Prade, U. Schröder, and W. Kress, Phys. Rev. B 43, 7, 5451 (1991). 